\title{
DERIVATION OF THE BIDOMAIN EQUATIONS FOR A BEATING HEART WITH A GENERAL MICROSTRUCTURE*
}

\author{
G. RICHARDSON ${ }^{\dagger}$ AND S. J. CHAPMAN ${ }^{\ddagger}$
}

\begin{abstract}
A novel multiple scales method is formulated that can be applied to problems which have an almost periodic microstructure not in Cartesian coordinates but in a general curvilinear coordinate system. The method is applied to a model of the electrical activity of cardiac myocytes and used to derive a version of the bidomain equations describing the macroscopic electrical activity of cardiac tissue. The treatment systematically accounts for the nonuniform orientation of the cells within the tissue and for deformations of the tissue occurring as a result of the heart beat.
\end{abstract}

Key words. multiple scales, homogenization, cardiac myocyte, conductivity, bidomain, deformation

AMS subject classifications. 35B27, 92C30, 78A57

DOI. $10.1137 / 090777165$

1. Introduction. The bidomain model for the propagation of cardiac action potentials was formulated in the late 1970s $[1,21,11,26]$ and is used to describe the evolution of the electrical potential through the cardiac tissue. On a microscopic scale action potentials (pulses in the transmembrane potential) occur as a result of the flow of particular species of ions through ion channels which span the cell membrane. This flow of ions, from the intracellular space to the extracellular space (or vice-versa), is accompanied by a current flow which leads to changes in the charge lying in the Debye layers on either side of the membrane and hence also to the transmembrane potential. The frequency of conformational changes of certain ion channels (i.e., the relative amount of time they spend open or closed) is affected by the transmembrane potential. Action potentials are initiated as a result of this feedback between transmembrane current flow, transmembrane potential, and changes in ion-channel conformation. In neurons they propagate as isolated pulses along axons (long thin structures branching from the cell body); see, for example, [13]. In contrast, action potentials in cardiac tissue propagate through a fully three-dimensional structure formed by an interconnecting array (or syncytium) of cardiac myocytes.

Cardiac myocytes have a long thin structure and align along a preferred direction within the cardiac tissue, thus giving this tissue its characteristic anisotropic macroscopic electrical and elastic properties. Furthermore the direction of orientation of the myocytes changes throughout the tissue and is a function of both space - as, for example, a transit is made across the ventricle wall - and time as the heart beats. (A two-dimensional caricature of the variable myocyte alignment is given in Figure 1.1.) The internal structure and myocyte orientation has been mapped, in a number of species, by diffusion tensor magnetic resonance imaging (see, for example, $[15,30]$ ). The propagation of action potentials through this highly complex tissue has been experimentally investigated using optical techniques which have been recently extended,

\footnotetext{
* Received by the editors November 16, 2009; accepted for publication (in revised form) January 26, 2011; published electronically May 4, 2011.

http://www.siam.org/journals/siap/71-3/77716.html

${ }^{\dagger}$ School of Mathematics, University of Southampton, Southampton SO17 1BJ, UK (G.Richardson @soton.ac.uk).

¥The Mathematical Institute, University of Oxford, 24-29 St Giles', Oxford OX1 3LB, UK (chapman@maths.ox.ac.uk).
} 


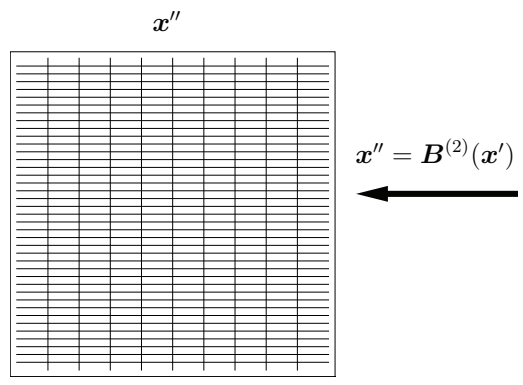

Reference Frame

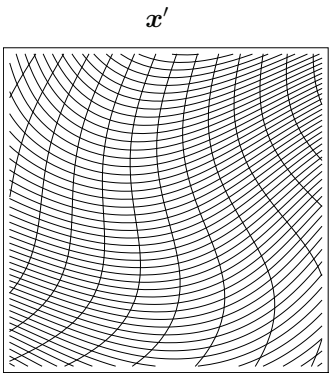

Lagrangian Frame

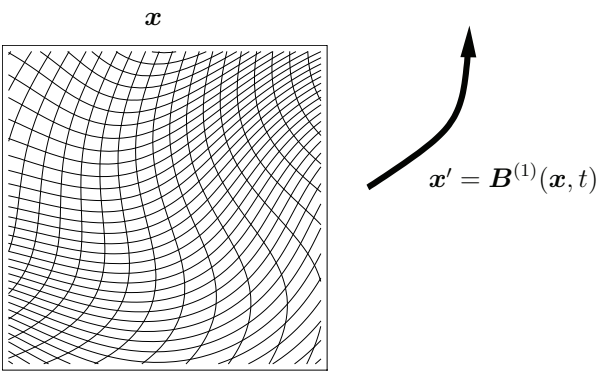

Eulerian frame

FIG. 1.1. An illustration of the three frames we use: the Eulerian laboratory frame, in which a point is defined by $\boldsymbol{x}$, its position with respect to a fixed origin; the Lagrangian frame, in which a point is defined by $\boldsymbol{x}^{\prime}$, its position in the resting heart; and the reference frame (with coordinates $\left.\boldsymbol{x}^{\prime \prime}\right)$.

by the use of optical coherence tomography, to provide three-dimensional maps of the propagation extending to a $2-3 \mathrm{~mm}$ depth (see [10] and references therein).

At various lengthscales different models apply to the phenomena underlying the propagation of action potentials. On the lengthscale of the membrane thickness, the electrochemistry of the ion solutions in the intra- and extracellular spaces can be described by the Poisson-Nernst-Planck equations (conservation equations for ion concentrations coupled to Poisson's equation for the electric field). A cell-scale model for action potential propagation is provided by assuming that current flow in the intra- and extracellular electrolytes is approximated by Ohm's law and charge conservation (this gives Laplace's equation for the electrical potential), that the normal component of electric current density is continuous across the membrane, and that the membrane's electrical properties can be described by a circuit consisting of a linear capacitor and nonlinear resistor connected in parallel (such as that shown in Figure 2.1 below). Transmembrane resistance is determined by the properties of the ion channels spanning the membrane and is usually described by a set of cell-specific phenomenological equations. Thus, for example, squid giant axons are well modelled by the Hodgkin-Huxley equations [13], whereas the behavior of the ventricular cardiac myocyte membrane is more complex, and a whole series of models (reviewed in [22]) have been formulated in order to better describe its electrical properties. These include the Beeler-Reuter equations [2] and the Luo-Rudy equations [20]. A formal derivation of the cell-scale model model from the Poisson-Nernst-Planck equations is made in [28].

In the case of cardiac tissue, which is formed from a three-dimensional array of interconnected myocytes, the cell-scale model is replaced by three-dimensional bi- 
domain equations on the tissue lengthscale. The one-dimensional versions of these bidomain equations can be used to determine the conduction velocity and to investigate restitution and collision of planar waves. Similarly the two-dimensional version of the equations can be used to investigate spiral waves and their drift (see, for example, [34]).

In [17] Krassowska and Neu use an asymptotic multiple-scales method to derive the bidomain equations from the cell-scale model, in tissue composed of a uniformly oriented periodic array of myocytes, a result which has subsequently been made rigorous in [25]. In reality myocytes in cardiac tissue are not uniformly oriented, and their long axis is aligned in different directions at different points in the tissue. Additionally, myocytes deform significantly as the heart beats, and a quantitative description of cardiac electrical activity needs to take this into account. We remark that the homogenization procedure is applicable only where the lengthscale for variations in the action potential, and that for variations in the myocyte orientation and density, are both much greater than the cell lengthscale. Estimates for the length and width of a cardiac myocyte range between $50-150 \mu \mathrm{m}$ and $15-25 \mu \mathrm{m}$, respectively, in humans $[18,27]$ and $100-120 \mu \mathrm{m}$ and $24-27 \mu \mathrm{m}$, respectively, in rats [4], while that for the thickness of the left ventricle (giving a typical tissue lengthscale) ranges between 12 $15 \mathrm{~mm}$ in humans $[23,32]$ and $1.5-2.7 \mathrm{~mm}$ in rats [19]. The typical lengthscale for variations in the action potential can be derived by multiplying the typical action potential velocity of $0.5 \mathrm{~ms}^{-1}[8]$ by the timescale for the passage of an action potential past a fixed point. Experimental graphs of the latter (timescale) [32] show that the sharp front of the action potential passes in about $5 \times 10^{-3}$ s while its smoother tail takes about $5 \times 10^{-2} \mathrm{~s}$ to pass, giving figures for the lengthscale of action potential variation between $2.5 \times 10^{-3} \mathrm{~m}$ and $2.5 \times 10^{-2} \mathrm{~m}$, both of which are very much bigger than the size of the myocyte. A lower figure for the lengthscale of the sharp front of the action potential, in the atrium of a dog, is given by [31] as $0.25-1 \mathrm{~mm}$; this is still significantly bigger than the cardiac myocyte.

An attempt to generalize the multiple-scales analysis of Krassowska and Neu to a more realistic tissue geometry has been made by Keener and Panfilov in the appendix of [16]. They suppose that heart tissue comprises an array of myocytes whose orientation is locally almost periodic but whose axis of orientation changes over the tissue scale. They introduce a set of curvilinear coordinates aligned with the fibers in the tissue, and suppose that in these coordinates the structure of the tissue is periodic. Thus the multiple-scales technique can be applied to the new equations in curvilinear coordinates. Finally, after the fast (cell) scale has been averaged out, the reverse coordinate transformation is applied to rewrite the tissue-scale equations (i.e., the bidomain equations) in Cartesian coordinates. It is assumed in [16] that the required coordinate transformation is a map that locally rotates coordinates without shearing or stretching them; unfortunately the only such map is a global rotation.

Here we aim to correct this error and formally derive the bidomain equations from the cell-scale model using a more general multiple-scales approach. Because in [16] the coordinate transformation is applied before the slow and fast scales are introduced, in effect both the slow and fast scales are transformed, with the result that the slow scale has to be untransformed at the end of the analysis. Here we will apply the coordinate transformation only to the fast scale; in effect we build the coordinate transformation into the multiple-scales ansatz in one step rather than two.

Thus we postulate the existence of a transformation from the time-dependent configuration of the heart to a regular "reference" frame (the domain of the curvilinear coordinates), which comprises a number of periodic boxes each containing one 


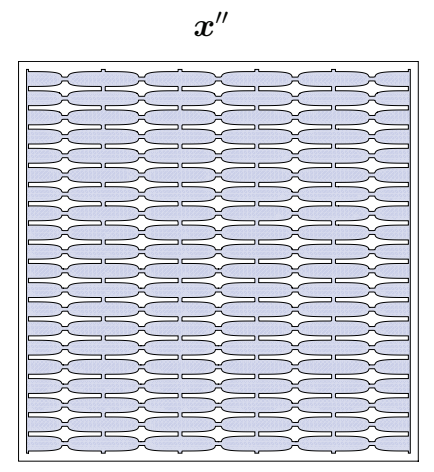

Reference Frame

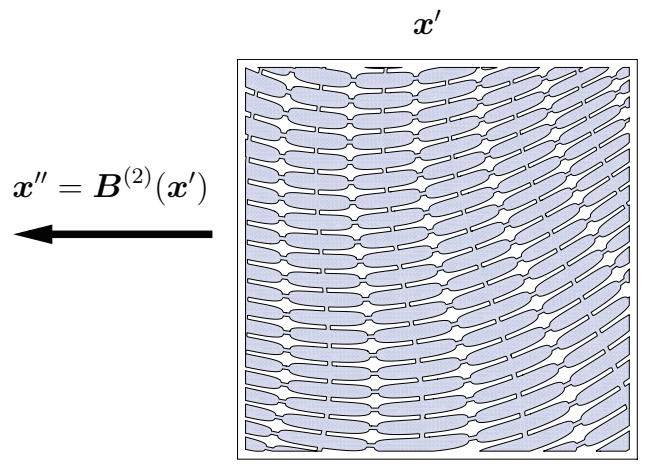

Lagrangian Frame

$\boldsymbol{x}$
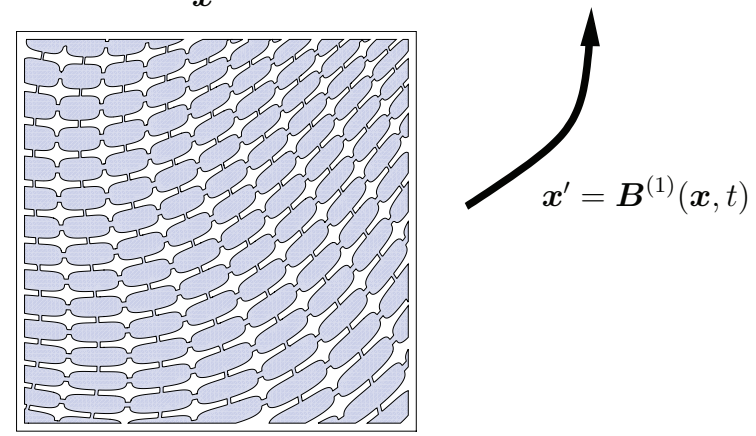

Eulerian frame

FIG. 1.2. An illustration of a typical cell geometry showing the elongated and interconnected myocytes in each of the three frames.

myocyte (or possibly a group of myocytes) and is thus amenable to attack by the method of multiple scales. We allow this transformation to be completely general, including shear and stretch (which we will see are unavoidable) as well as rotation (see Figure 1.1). This will also allow us to consider the effect of time-dependent transformations. The results we obtain are thus applicable not only to scenarios such as pacing and defibrillation, in which the tissue does not move much, but also to the heart beat, in which significant time-dependent deformation of the tissue occurs.

It is helpful to specify a point within the heart in terms of its position $\boldsymbol{x}$ measured with respect to a fixed origin (the Eulerian frame), in terms of its position $\boldsymbol{x}^{\prime}$ in the resting heart (the Lagrangian frame), and in terms of its position $\boldsymbol{x}^{\prime \prime}$ in the reference frame (see Figures 1.1 and 1.2). These vectors are related by the transformations

$$
\boldsymbol{x}^{\prime}=\boldsymbol{B}^{(1)}(\boldsymbol{x}, t) \quad \text { and } \quad \boldsymbol{x}^{\prime \prime}=\boldsymbol{B}^{(2)}\left(\boldsymbol{x}^{\prime}\right),
$$

so that the map from position vectors $\boldsymbol{x}$ in the Eulerian frame to those in the reference frame $\boldsymbol{x}^{\prime \prime}$ is given by the composition of the above transformations

$$
\boldsymbol{x}^{\prime \prime}=\boldsymbol{B}(\boldsymbol{x}, t), \quad \text { where } \boldsymbol{B}(\boldsymbol{x}, t)=\boldsymbol{B}^{(2)}\left(\boldsymbol{B}^{(1)}(\boldsymbol{x}, t)\right) .
$$

Despite the lack of a formal derivation of the bidomain equations in nonuniformly oriented tissue, there are a number of works (e.g., [24, 29]) which simulate inhomogeneous cardiac tissue using the bidomain model. In these the conductivity tensor 
is usually assumed to be diagonal in a local frame and aligned with the heart fibers, with one conductivity along the fiber direction and another conductivity orthogonal to the fiber direction. These "axial" and "transverse" conductivities are usually determined experimentally rather than via a homogenization procedure. The analysis which follows will examine the validity of these assumptions and give an indication of how the conductivity tensor might be altered by the deformation of the tissue during a heart beat.

Rigorous results on the existence, uniqueness, and regularity of solutions to the bidomain model have been derived in $[3,33]$.

2. The model. We will start by writing down the model in terms of physical coordinates in the standard Eulerian frame.

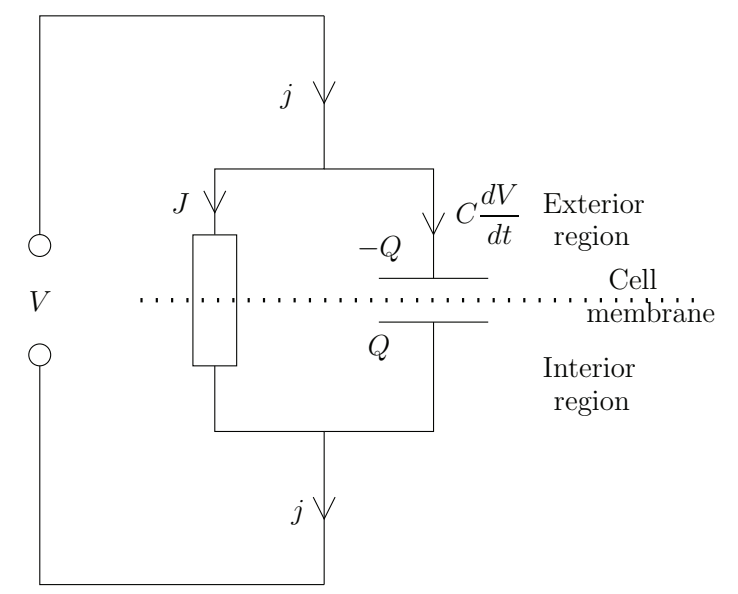

FIG. 2.1. Illustration of the electrical circuit used to describe the membrane's electrical properties, comprising a resistor and a capacitor in parallel.

The membrane (and its Debye layers) act as an electrical capacitor, with the ability to store charge. Furthermore current can pass through arrays of ion channels located in the membrane (which have a nonlinear current voltage relationship primarily as a consequence of channel gating) so that the membrane and Debye layers can be modelled, locally, as a nonlinear resistor in parallel with a capacitor (see Figure 2.1). Globally the membrane is modelled by defining a nonlinear current density-voltage relation $J(V, t)$ for the resistive flow of current across the membrane, from the exterior domain to the interior, through the ion channels (see, e.g., [22]). Here $V$ is the potential difference between the exterior region and the interior one (i.e., $V=V^{(e x)}-V^{(i n)}$ ); thus $V$ is minus the standard transmembrane potential, which is the potential difference between the interior and the exterior. ${ }^{1}$ In addition, the capacitative properties of the membrane are modelled as a linear capacitor with capacitance $\mathcal{C}$ per unit area, so that the current density flowing through the capacitor is $\mathcal{C} \frac{d V}{d t}$. Kirchoff's law states that the total current density flowing through the membrane $j$ into the cell is the sum of the resistive and capacitative components, so that

$$
j=\mathcal{C} \frac{d V}{d t}+J(V, t)
$$

\footnotetext{
${ }^{1}$ We define $V$ this way around so that positive $V$ is associated with a positive transmembrane current $J$. In turn, $J$ is defined to be positive where current flows from the exterior to the interior of the membrane, in line with [13].
} 
The electrolytes on either side of the membrane behave as Ohmic conductors, so that their electrical properties are described by

$$
\begin{aligned}
\boldsymbol{j}^{(e x)}=-\varsigma^{(e x)} \nabla \phi^{(e x)}, & \nabla \cdot \boldsymbol{j}^{(e x)}=0, \\
\boldsymbol{j}^{(i n)}=-\varsigma^{(i n)} \nabla \phi^{(i n)}, & \nabla \cdot \boldsymbol{j}^{(i n)}=0,
\end{aligned}
$$

where $j$ is the current density, $\phi$ is the electric potential, and $\varsigma$ is the electrical resistivity of the electrolyte. Since the net charge on the capacitor is zero, current flow normal to the membrane must be continuous across the membrane, so that

$$
\left.\boldsymbol{j}^{(i n)} \cdot \boldsymbol{n}\right|_{\partial \Omega}=\left.\boldsymbol{j}^{(e x)} \cdot \boldsymbol{n}\right|_{\partial \Omega}=-j,
$$

where the superscript (in) denotes the interior region, $(e x)$ denotes the exterior region, and $\boldsymbol{n}$ is the unit normal to the membrane surface pointing away from the interior region.

Combining these equations results in the following model for the potentials $\phi^{(i n)}$ and $\phi^{(e x)}$ in the interior and exterior regions, respectively:

$$
\begin{array}{rr}
\nabla^{2} \phi^{(e x)}=0 & \text { in } \Omega_{e}(t), \\
\nabla^{2} \phi^{(i n)}=0 & \text { in } \Omega_{i}(t), \\
\left.\varsigma^{(e x)}(\boldsymbol{n} \cdot \nabla) \phi^{(e x)}\right|_{\partial \Omega(t)}=\left.\varsigma^{(i n)}(\boldsymbol{n} \cdot \nabla) \phi^{(i n)}\right|_{\partial \Omega(t)}, \\
\phi^{(e x)}-\left.\phi^{(i n)}\right|_{\partial \Omega(t)}=V, \\
-\mathcal{C} \frac{\mathrm{d} V}{\mathrm{~d} t}=J(V, t)-\left.\varsigma^{(i n)}(\boldsymbol{n} \cdot \nabla) \phi^{(i n)}\right|_{\partial \Omega(t)},
\end{array}
$$

where we note that $\phi^{(e x)}$ and $\phi^{(i n)}$ are defined only up to the addition of the (same) arbitrary constant. A formal derivation of the model from the underlying PoissonNernst-Planck equations is made in [28].

Nondimensionalization. We nondimensionalize using the following scales:

$\phi=\Phi_{0} \hat{\phi}, \quad V=\Phi_{0} \hat{V}, \quad t=\tau \hat{t}, \quad x=L \hat{\boldsymbol{x}}, \quad \varsigma^{(e x)}=\varsigma_{0} \hat{\varsigma}^{(e x)}, \quad \varsigma^{(i n)}=\varsigma_{0} \hat{\varsigma}^{(i n)}, \quad J=J_{0} \hat{J}$,

where $\Phi_{0}$ is the typical potential drop across the membrane (and could, for example, sensibly be chosen equal to the modulus of the resting membrane potential of $90 \mathrm{mV}$ ), $L$ is the typical lengthscale of the cardiac tissue (rather than that of an individual myocyte), $\varsigma_{0}$ is the typical conductivity of the electrolytes, and $J_{0}$ is the typical current density passing through the ion channels spanning the membrane. The result of this nondimensionalization is the following dimensionless model:

$$
\begin{array}{rr}
\hat{\nabla}^{2} \hat{\phi}^{(e x)}=0 & \text { in } \Omega_{e}, \\
\hat{\nabla}^{2} \hat{\phi}^{(i n)}=0 & \text { in } \Omega_{i}, \\
\left.\hat{\varsigma}^{(e x)}(\boldsymbol{n} \cdot \hat{\nabla}) \hat{\phi}^{(e x)}\right|_{\partial \Omega}=\left.\hat{\varsigma}^{(i n)}(\boldsymbol{n} \cdot \hat{\nabla}) \hat{\phi}^{(i n)}\right|_{\partial \Omega,} \\
\hat{\phi}^{(e x)}-\left.\hat{\phi}^{(i n)}\right|_{\partial \Omega}=\hat{V}, \\
-C \frac{\mathrm{d} \hat{V}}{\mathrm{~d} \hat{t}}=\hat{J}(\hat{V}, \hat{t})-\left.Z \hat{\varsigma}^{(i n)}(\boldsymbol{n} \cdot \hat{\nabla}) \hat{\phi}^{(i n)}\right|_{\partial \Omega,}
\end{array}
$$

in which the dimensionless parameters are defined by

$$
Z=\frac{\varsigma_{0} \Phi_{0}}{J_{0} L}, \quad C=\frac{\mathcal{C} \Phi_{0}}{\tau J_{0}}
$$


A further dimensionless parameter of importance is $\epsilon$, which gives the ratio of the typical lengthscale of cardiac myocytes to that of the cardiac tissue and is thus small (see the discussion in section 1 ). In the following we shall investigate the distinguished asymptotic limit in which $C=O(1)$ and $Z=O(1 / \epsilon)$, which is of direct relevance to the action of cardiac muscle.

Henceforth we drop the hats from the dimensionless variables.

3. Derivation of the bidomain equations. Given the large number of cardiac myocytes in the heart, it is extremely expensive to solve the model (2.6)-(2.10) directly. In this section we use the method of multiple scales to derive an averaged (or homogenized) model for the potential, valid over the scale of many myocytes, in the distinguished limit $\epsilon \ll 1$, with $C=O(1)$ and $Z=O(1 / \epsilon)$. The analysis is complicated by the facts that (i) the elongated cardiac myocytes are not uniformly oriented at all positions within the heart and (ii) the heart tissue undergoes significant deformations in the course of the cardiac cycle.

3.1. Coordinates. As mentioned in the introduction, in order to use the method of multiple scales on this problem we introduce a transformation which maps the cardiac myocyte domains (at a general stage in the cardiac cycle) onto a periodic lattice (see Figures 1.1 and 1.2). Here we consider each box on the lattice to contain one myocyte, and the function $\boldsymbol{B}$ to map the actual configuration (as observed in the real heart tissue at some particular time) to a periodic reference configuration in which each myocyte occupies a box of the same size and geometry. We formalize this transformation by defining $\boldsymbol{x}$ to be a macroscale variable in the real configuration, which measures distances over many myocytes, and $\boldsymbol{y}^{\prime \prime}=\boldsymbol{x}^{\prime \prime} / \epsilon$ to be a microscale variable in the periodic reference configuration, which measures distances over the scale of a single myocyte. These variables are related by

$$
\boldsymbol{y}^{\prime \prime}=\frac{1}{\epsilon} \boldsymbol{B}(\boldsymbol{x}, t)
$$

Although they do not present it this way, Keener and Panfilov effectively look for solutions which are functions of $\boldsymbol{B}(\boldsymbol{x}, t)$ and $\boldsymbol{B}(\boldsymbol{x}, t) / \epsilon$, imposing the condition that this solution is periodic in $\boldsymbol{B}(\boldsymbol{x}, t) / \epsilon$. Having derived the homogenized equations for the leading-order solution as a function of $\boldsymbol{B}(\boldsymbol{x}, t)$, they then need to undo the coordinate transformation to write the equations in terms of $\boldsymbol{x}$.

In fact, since the multiple scales technique considers the slow and fast scales to be independent, there is no need to transform the slow variable. Instead we look for solutions which are functions of the original macroscale variable $\boldsymbol{x}$ and the transformed microscale variable $\boldsymbol{y}^{\prime \prime}$, imposing periodicity in $\boldsymbol{y}^{\prime \prime}$.

Spatial derivatives in (2.6)-(2.10) transform according to

$$
\frac{\partial}{\partial x_{i}} \longrightarrow \frac{\partial}{\partial x_{i}}+\frac{1}{\epsilon} F_{i j} \frac{\partial}{\partial y_{j}^{\prime \prime}}
$$

where $x_{i}$ and $y_{i}^{\prime \prime}$ represent the components of $\boldsymbol{x}$ and $\boldsymbol{y}^{\prime \prime}$, respectively, in the $i$ th direction and

$$
F_{i j}=\frac{\partial B_{j}}{\partial x_{i}}
$$

At this stage it is also helpful to introduce some additional notation to distinguish the boundaries of the domains $\Omega_{i}^{\prime \prime}$ and $\Omega_{e}^{\prime \prime}$ in the unit cell. As usual we denote the 
(I)

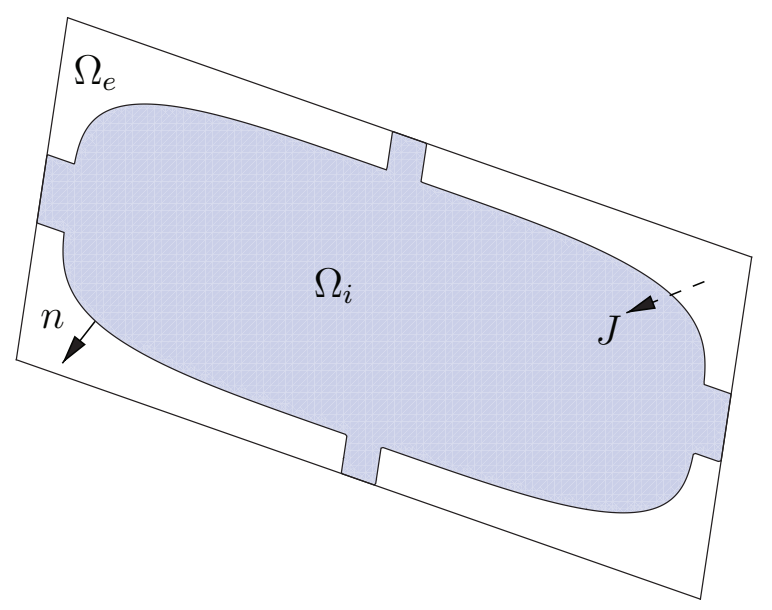

(II)

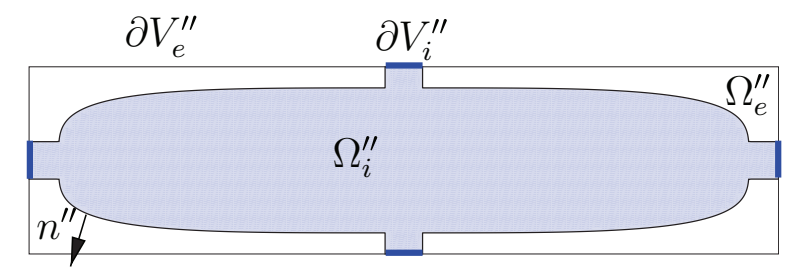

FIG. 3.1. The geometry of the unit cell in (I) the Eulerian frame and (II) the reference frame. The intracellular and extracellular domains are indicated, along with the transmembrane current $J$ and the normals $\boldsymbol{n}$ and $\boldsymbol{n}^{\prime \prime}$.

common boundary between $\Omega_{i}^{\prime \prime}$ and $\Omega_{e}^{\prime \prime}$ as $\partial \Omega^{\prime \prime}$. The boundary of the unit cell which lies in $\Omega_{i}^{\prime \prime}$ we denote by $\partial V_{i}^{\prime \prime}$, while that which lies in $\Omega_{e}^{\prime \prime}$ we denote by $\partial V_{e}^{\prime \prime}$. The unit cells in the Eulerian frame and the reference frame are illustrated in Figure 3.1.

Before we can proceed with the asymptotic expansion of the solution we need to work out how to transform the derivative

$$
\frac{\partial}{\partial n} \equiv \boldsymbol{n} \cdot \nabla
$$

into multiple scales. To do this we need to determine how the normal $\boldsymbol{n}^{\prime \prime}$ in the unit cell reference coordinates relates to the normal $\boldsymbol{n}$ in the real (Eulerian) coordinates.

Suppose that the boundary to a particular myocyte is given, in the reference frame, by the functional relation $\psi\left(\boldsymbol{y}^{\prime \prime}\right)=0$. The normal to this boundary $\boldsymbol{n}^{\prime \prime}$ (again in the reference frame) is then given by

$$
\boldsymbol{n}^{\prime \prime}=\frac{\nabla^{\prime \prime} \psi}{\left|\nabla^{\prime \prime} \psi\right|}
$$

where $\nabla^{\prime \prime}$ is the vector derivative with respect to the $\boldsymbol{y}^{\prime \prime}$ variable. The normal in the reference frame is, of course, not equal to the normal $\boldsymbol{n}$ in the Eulerian frame. However, we can relate the two normals by transforming variables from $\boldsymbol{y}^{\prime \prime}$ to $\boldsymbol{x}$ in the function $\psi$ and noting that

$$
\boldsymbol{n}=\frac{\nabla \psi}{|\nabla \psi|},
$$


where here $\nabla$ is the vector derivative with respect to $\boldsymbol{x}$. The transformation of variables implies that

$$
\frac{\partial \psi}{\partial x_{i}}=\frac{1}{\epsilon} F_{i j} \frac{\partial \psi}{\partial y_{j}^{\prime \prime}}
$$

and hence that

$$
\boldsymbol{n}=\frac{F_{i j} \frac{\partial \psi}{\partial y_{j}^{\prime \prime}}}{\left(F_{m k} F_{m l} \frac{\partial \psi}{\partial y_{l}^{\prime \prime}} \frac{\partial \psi}{\partial y_{k}^{\prime \prime}}\right)^{1 / 2}} \boldsymbol{e}_{i}, \quad \text { whereas } \quad \boldsymbol{n}^{\prime \prime}=\frac{\frac{\partial \psi}{\partial y_{i}^{\prime \prime}}}{\left(\frac{\partial \psi}{\partial y_{m}^{\prime \prime}} \frac{\partial \psi}{\partial y_{m}^{\prime \prime}}\right)^{1 / 2}} \boldsymbol{e}_{i} .
$$

Here $\boldsymbol{e}_{i}$ is the unit vector in the direction of the $i$ th coordinate, and we use the Einstein summation convention. It follows that the components of $\boldsymbol{n}$ and $\boldsymbol{n}^{\prime \prime}$ are related by

$$
n_{i}=F_{i j} n_{j}^{\prime \prime} \frac{\left(\frac{\partial \psi}{\partial y_{m}^{\prime \prime}} \frac{\partial \psi}{\partial y_{m}^{\prime \prime}}\right)^{1 / 2}}{\left(F_{p q} F_{p r} \frac{\partial \psi}{\partial y_{q}^{\prime \prime}} \frac{\partial \psi}{\partial y_{r}^{\prime \prime}}\right)^{1 / 2}}
$$

and hence that

$$
n_{i}=F_{i j} n_{j}^{\prime \prime} \frac{1}{\left(F_{p q} F_{p r} n_{q}^{\prime \prime} n_{r}^{\prime \prime}\right)^{1 / 2}}
$$

We note that in the special case where $F$ is a rotation matrix it is also an orthogonal matrix and so has the property $F F^{T}=F^{T} F=I$ (in component notation, $F_{i k} F_{j k}=\delta_{i j}$ and $\left.F_{k i} F_{k j}=\delta_{i j}\right)$. From this it follows that (3.4) simplifies to

$$
n_{i}=F_{i j} n_{j}^{\prime \prime}
$$

In other words, the normal in the Eulerian frame is just the normal in the reference frame rotated by the matrix $F$.

3.2. The multiple-scales expansion. We look for a solution to (2.6)-(2.10) of the form $\phi=\phi\left(\boldsymbol{y}^{\prime \prime}, \boldsymbol{x}\right)$, which is periodic in $\boldsymbol{y}^{\prime \prime}$. We start by writing (2.6)-(2.10) in terms of the multiple-scales transformation of the derivatives (3.2) using (3.4) to rewrite (2.10) in terms of $\boldsymbol{n}^{\prime \prime}$. On writing $Z=\Theta / \epsilon$, where $\Theta$ is an $O(1)$ parameter, this gives

$$
\frac{\partial^{2} \phi^{(i n)}}{\partial x_{i}^{2}}+\frac{1}{\epsilon}\left(F_{i j} \frac{\partial^{2} \phi^{(i n)}}{\partial x_{i} \partial y_{j}^{\prime \prime}}+\frac{\partial}{\partial x_{i}}\left(F_{i j} \frac{\partial \phi^{(i n)}}{\partial y_{j}^{\prime \prime}}\right)\right)+\frac{1}{\epsilon^{2}} F_{i j} F_{i k} \frac{\partial^{2} \phi^{(i n)}}{\partial y_{j}^{\prime \prime} \partial y_{k}^{\prime \prime}}=0 \quad \text { for } \boldsymbol{y}^{\prime \prime} \in \Omega_{i}^{\prime \prime},
$$

$\frac{\partial^{2} \phi^{(e x)}}{\partial x_{i}^{2}}+\frac{1}{\epsilon}\left(F_{i j} \frac{\partial^{2} \phi^{(e x)}}{\partial x_{i} \partial y_{j}^{\prime \prime}}+\frac{\partial}{\partial x_{i}}\left(F_{i j} \frac{\partial \phi^{(e x)}}{\partial y_{j}^{\prime \prime}}\right)\right)+\frac{1}{\epsilon^{2}} F_{i j} F_{i k} \frac{\partial^{2} \phi^{(e x)}}{\partial y_{j}^{\prime \prime} \partial y_{k}^{\prime \prime}}=0 \quad$ for $\boldsymbol{y}^{\prime \prime} \in \Omega_{e}^{\prime \prime}$,

$$
\begin{aligned}
& \left.\varsigma^{(e x)} F_{p m} n_{m}^{\prime \prime}\left(\frac{\partial \phi^{(e x)}}{\partial x_{p}}+\frac{1}{\epsilon} F_{p q} \frac{\partial \phi^{(e x)}}{\partial y_{q}^{\prime \prime}}\right)\right|_{\partial \Omega^{\prime \prime}}=\left.\varsigma^{(i n)} F_{p m} n_{m}^{\prime \prime}\left(\frac{\partial \phi^{(i n)}}{\partial x_{p}}+\frac{1}{\epsilon} F_{p q} \frac{\partial \phi^{(i n)}}{\partial y_{q}^{\prime \prime}}\right)\right|_{\partial \Omega^{\prime \prime}}, \\
& (3.8) \quad \phi^{(e x)}-\left.\phi^{(i n)}\right|_{\partial \Omega^{\prime \prime}}=V, \\
& (3.9) \quad-\epsilon^{2} C \frac{\mathrm{d} V}{\mathrm{~d} t}=\epsilon^{2} J-\left.\Theta \varsigma^{(i n)}\left(F_{p q} \frac{\partial \phi^{(i n)}}{\partial y_{q}^{\prime \prime}}+\epsilon \frac{\partial \phi^{(i n)}}{\partial x_{p}}\right) \frac{F_{p m} n_{m}^{\prime \prime}}{\left(F_{l r} F_{l s} n_{r}^{\prime \prime} n_{s}^{\prime \prime}\right)^{1 / 2}}\right|_{\partial \Omega^{\prime \prime}} .
\end{aligned}
$$


We now look for an asymptotic solution to (3.5)-(3.9) in powers of $\epsilon$ of the form

$$
\begin{aligned}
\phi^{(i n)} & =\phi_{0}^{(i n)}(\boldsymbol{x}, t)+\epsilon \phi_{1}^{(i n)}\left(\boldsymbol{y}^{\prime \prime}, \boldsymbol{x}, t\right)+\epsilon^{2} \phi_{2}^{(i n)}\left(\boldsymbol{y}^{\prime \prime}, \boldsymbol{x}, t\right)+\cdots, \\
\phi^{(e x)} & =\phi_{0}^{(e x)}(\boldsymbol{x}, t)+\epsilon \phi_{1}^{(e x)}\left(\boldsymbol{y}^{\prime \prime}, \boldsymbol{x}, t\right)+\epsilon^{2} \phi_{2}^{(e x)}\left(\boldsymbol{y}^{\prime \prime}, \boldsymbol{x}, t\right)+\cdots, \\
V & =V_{0}(\boldsymbol{x}, t)+\cdots, \\
J & =J_{0}+\cdots .
\end{aligned}
$$

Now, in the system (3.5)-(3.9) the potential drop $V$ is defined only on the membrane (see (3.8)). However, the potential $V_{0}$ in (3.12) is a continuous function of (slow) space and time. When the domain itself is moving (as in a heartbeat) we must be careful to replace the total derivative of $V$ with the convective derivative of $V_{0}$, which we write as

$$
\frac{\mathrm{D} V_{0}}{\mathrm{D} t}=\frac{\partial V_{0}}{\partial t}+(\boldsymbol{U} \cdot \nabla) V_{0}
$$

where $\boldsymbol{U}$ is the tissue velocity.

The first-order problem for $\phi$. After substituting (3.10)-(3.13) into (3.5)-(3.9), we find that the leading-order equations are satisfied trivially due to our assumption that $\phi_{0}^{(i n)}$ and $\phi_{0}^{(e x)}$ are independent of $\boldsymbol{y}^{\prime \prime}$. At first order in $\epsilon$ we find the following linear system for $\phi_{1}^{(i n)}$ :

$$
\begin{gathered}
F_{i j} F_{i k} \frac{\partial^{2} \phi_{1}^{(i n)}}{\partial y_{j}^{\prime \prime} \partial y_{k}^{\prime \prime}}=0 \quad \text { for } \boldsymbol{y}^{\prime \prime} \in \Omega_{i}^{\prime \prime} \\
\left.F_{i j} F_{i k} n_{j}^{\prime \prime} \frac{\partial \phi_{1}^{(i n)}}{\partial y_{k}^{\prime \prime}}\right|_{\partial \Omega^{\prime \prime}}=-F_{i j} n_{j}^{\prime \prime} \frac{\partial \phi_{0}^{(i n)}}{\partial x_{i}} \\
\phi_{1}^{(i n)} \quad \text { periodic in } \boldsymbol{y}^{\prime \prime}
\end{gathered}
$$

In a similar fashion we can write down an almost identical problem for $\phi_{1}^{(e x)}$ using (3.6), (3.7), and (3.9); this is

$$
\begin{gathered}
F_{i j} F_{i k} \frac{\partial^{2} \phi_{1}^{(e x)}}{\partial y_{j}^{\prime \prime} \partial y_{k}^{\prime \prime}}=0 \quad \text { for } \boldsymbol{y}^{\prime \prime} \in \Omega_{e}^{\prime \prime} \\
\left.F_{i j} F_{i k} n_{j}^{\prime \prime} \frac{\partial \phi_{1}^{(e x)}}{\partial y_{k}^{\prime \prime}}\right|_{\partial \Omega^{\prime \prime}}=-F_{i j} n_{j}^{\prime \prime} \frac{\partial \phi_{0}^{(e x)}}{\partial x_{i}} \\
\phi_{1}^{(e x)} \quad \text { periodic in } \boldsymbol{y}^{\prime \prime} .
\end{gathered}
$$

Recall that $\boldsymbol{n}^{\prime \prime}$ is the outward normal to $\Omega_{i}^{\prime \prime}$ and the inward normal to $\Omega_{e}^{\prime \prime}$.

A solvability condition. Integrating the divergence of an arbitrary vector function $T^{(i n)}$ over the domain $\Omega_{i}^{\prime \prime}$ and applying the divergence theorem gives

$$
\int_{\Omega_{i}^{\prime \prime}} \frac{\partial T_{j}^{(i n)}}{\partial y_{j}^{\prime \prime}} d V^{\prime \prime}=\int_{\partial \Omega^{\prime \prime}} T_{j}^{(i n)} n_{j}^{\prime \prime} d S^{\prime \prime}+\int_{\partial V_{i}^{\prime \prime}} T_{j}^{(i n)} n_{j}^{\prime \prime} d S^{\prime \prime} .
$$

If $\boldsymbol{T}^{(i n)}$ is periodic in $\boldsymbol{y}^{\prime \prime}$, the last term is identically zero, so that

$$
\int_{\Omega_{i}^{\prime \prime}} \frac{\partial T_{j}^{(i n)}}{\partial y_{j}^{\prime \prime}} d V^{\prime \prime}=\int_{\partial \Omega^{\prime \prime}} T_{j}^{(i n)} n_{j}^{\prime \prime} d S^{\prime \prime} .
$$


Equivalently, (for a periodic vector $\boldsymbol{T}^{(\boldsymbol{e x})}$ ) we can write (on noting that $\boldsymbol{n}^{\prime \prime}$ is the inward normal to $\Omega_{e}^{\prime \prime}$ )

$$
\int_{\Omega_{e}^{\prime \prime}} \frac{\partial T_{j}^{(e x)}}{\partial y_{j}^{\prime \prime}} d V^{\prime \prime}=-\int_{\partial \Omega^{\prime \prime}} T_{j}^{(e x)} n_{j}^{\prime \prime} d S^{\prime \prime}
$$

Now, problems (3.14)-(3.15) can be expressed in the form

$$
\begin{aligned}
& \frac{\partial T_{j}^{(i n)}}{\partial y_{j}^{\prime \prime}}=0 \quad \text { for } \boldsymbol{y}^{\prime \prime} \in \Omega_{i}^{\prime \prime},\left.\quad T_{j}^{(i n)} n_{j}^{\prime \prime}\right|_{\partial \Omega^{\prime \prime}}=0, \\
& \frac{\partial T_{j}^{(e x)}}{\partial y_{j}^{\prime \prime}}=0 \quad \text { for } \boldsymbol{y}^{\prime \prime} \in \Omega_{e}^{\prime \prime},\left.\quad T_{j}^{(e x)} n_{j}^{\prime \prime}\right|_{\partial \Omega^{\prime \prime}}=0 \text {, }
\end{aligned}
$$

with $\boldsymbol{T}^{(i n)}$ and $\boldsymbol{T}^{(\boldsymbol{e x})}$ periodic in $\boldsymbol{y}^{\prime \prime}$, where

$$
T_{j}^{(i n)}=F_{i j}\left(F_{i k} \frac{\partial \phi_{1}^{(i n)}}{\partial y_{k}^{\prime \prime}}+\frac{\partial \phi_{0}^{(i n)}}{\partial x_{i}}\right), \quad T_{j}^{(e x)}=F_{i j}\left(F_{i k} \frac{\partial \phi_{1}^{(e x)}}{\partial y_{k}^{\prime \prime}}+\frac{\partial \phi_{0}^{(e x)}}{\partial x_{i}}\right)
$$

Substituting (3.18a) and (3.18b) into the solvability conditions (3.16) and (3.17), respectively, we see that these solvability conditions are satisfied trivially. At the next order the corresponding solvability conditions will result in the bidomain equations.

We can write the solutions to (3.14) and (3.15) as

$$
\begin{aligned}
& \phi_{1}^{(i n)}=\sum_{r=1}^{3} \frac{\partial \phi_{0}^{(i n)}}{\partial x_{r}} \Xi_{r}^{(i n)}\left(\boldsymbol{y}^{\prime \prime}\right)+A^{(i n)}(\boldsymbol{x}, t), \\
& \phi_{1}^{(e x)}=\sum_{r=1}^{3} \frac{\partial \phi_{0}^{(e x)}}{\partial x_{r}} \Xi_{r}^{(e x)}\left(\boldsymbol{y}^{\prime \prime}\right)+A^{(e x)}(\boldsymbol{x}, t),
\end{aligned}
$$

where $A^{(i n)}(\boldsymbol{x}, t)$ and $A^{(e x)}(\boldsymbol{x}, t)$ are arbitrary functions of integration and the functions $\Xi_{r}^{(i n)}\left(\boldsymbol{y}^{\prime \prime}\right)$ and $\Xi_{r}^{(e x)}\left(\boldsymbol{y}^{\prime \prime}\right)(r=1,2,3)$ satisfy the cell problems

$$
F_{i j} F_{i k} \frac{\partial^{2} \Xi_{r}^{(i n)}}{\partial y_{j}^{\prime \prime} \partial y_{k}^{\prime \prime}}=0 \quad \text { for } \boldsymbol{y}^{\prime \prime} \in \Omega_{i}^{\prime \prime},\left.\quad F_{i j} F_{i k} n_{j}^{\prime \prime} \frac{\partial \Xi_{r}^{(i n)}}{\partial y_{k}^{\prime \prime}}\right|_{\partial \Omega^{\prime \prime}}=-F_{r q} n_{q}^{\prime \prime},
$$

$$
\Xi_{r}^{(i n)} \quad \text { periodic in } \boldsymbol{y}^{\prime \prime}
$$

and

$$
\begin{gathered}
F_{i j} F_{i k} \frac{\partial^{2} \Xi_{r}^{(e x)}}{\partial y_{j}^{\prime \prime} \partial y_{k}^{\prime \prime}}=0 \quad \text { for } \boldsymbol{y}^{\prime \prime} \in \Omega_{e}^{\prime \prime},\left.\quad F_{i j} F_{i k} n_{j}^{\prime \prime} \frac{\partial \Xi_{r}^{(e x)}}{\partial y_{k}^{\prime \prime}}\right|_{\partial \Omega^{\prime \prime}}=-F_{r q} n_{q}^{\prime \prime}, \\
\Xi_{r}^{(e x)} \quad \text { periodic in } \boldsymbol{y}^{\prime \prime} .
\end{gathered}
$$

The second-order problem for $\phi$. Proceeding to second order in the expansion of 
(3.5)-(3.9) leads to the following problem for $\phi_{2}^{(i n)}$ and $\phi_{2}^{(e x)}$ :

$$
F_{i j} F_{i k} \frac{\partial^{2} \phi_{2}^{(i n)}}{\partial y_{j}^{\prime \prime} \partial y_{k}^{\prime \prime}}+2 F_{i j} \frac{\partial^{2} \phi_{1}^{(i n)}}{\partial x_{i} \partial y_{j}^{\prime \prime}}+\frac{\partial F_{i j}}{\partial x_{i}} \frac{\partial \phi_{1}^{(i n)}}{\partial y_{j}^{\prime \prime}}+\frac{\partial^{2} \phi_{0}^{(i n)}}{\partial x_{i}^{2}}=0 \quad \text { for } \boldsymbol{y}^{\prime \prime} \in \Omega_{i},
$$

$$
\left.\Theta \varsigma^{(i n)}\left(F_{i j} F_{i k} n_{j}^{\prime \prime} \frac{\partial \phi_{2}^{(i n)}}{\partial y_{k}^{\prime \prime}}+F_{i j} n_{j}^{\prime \prime} \frac{\partial \phi_{1}^{(i n)}}{\partial x_{i}}\right)\right|_{\partial \Omega^{\prime \prime}}=\left(F_{l r} F_{l s} n_{r}^{\prime \prime} n_{s}^{\prime \prime}\right)^{1 / 2}\left(J_{0}+C \frac{\mathrm{D} V_{0}}{\mathrm{D} t}\right),
$$

$$
F_{i j} F_{i k} \frac{\partial^{2} \phi_{2}^{(e x)}}{\partial y_{j}^{\prime \prime} \partial y_{k}^{\prime \prime}}+2 F_{i j} \frac{\partial^{2} \phi_{1}^{(e x)}}{\partial x_{i} \partial y_{j}^{\prime \prime}}+\frac{\partial F_{i j}}{\partial x_{i}} \frac{\partial \phi_{1}^{(e x)}}{\partial y_{j}^{\prime \prime}}+\frac{\partial^{2} \phi_{0}^{(e x)}}{\partial x_{i}^{2}}=0 \quad \text { for } \boldsymbol{y}^{\prime \prime} \in \Omega_{e},
$$

$$
\begin{aligned}
& \left.\Theta \varsigma^{(e x)}\left(F_{i j} F_{i k} n_{j}^{\prime \prime} \frac{\partial \phi_{2}^{(e x)}}{\partial y_{k}^{\prime \prime}}+F_{i j} n_{j}^{\prime \prime} \frac{\partial \phi_{1}^{(e x)}}{\partial x_{i}}\right)\right|_{\partial \Omega^{\prime \prime}}=\left(F_{l r} F_{l s} n_{r}^{\prime \prime} n_{s}^{\prime \prime}\right)^{1 / 2}\left(J_{0}+C \frac{\mathrm{D} V_{0}}{\mathrm{D} t}\right), \\
& 3.26) \quad \phi_{0}^{(e x)}-\left.\phi_{0}^{(i n)}\right|_{\partial \Omega^{\prime \prime}}=V_{0},
\end{aligned}
$$

with $\phi_{2}^{(i n)}$ and $\phi_{2}^{(e x)}$ periodic in $\boldsymbol{y}^{\prime \prime}$.

A solvability condition on the second-order problem. Equations (3.22)-(3.25) can be rewritten in the form

$$
\begin{aligned}
\frac{\partial T_{j}^{(i n)}}{\partial y_{j}^{\prime \prime}} & =-\frac{\partial}{\partial x_{i}}\left(F_{i j} \frac{\partial \phi_{1}^{(i n)}}{\partial y_{j}^{\prime \prime}}+\frac{\partial \phi_{0}^{(i n)}}{\partial x_{i}}\right) \text { for } \boldsymbol{y}^{\prime \prime} \in \Omega_{i}, \\
\left.\Theta \varsigma^{(i n)} T_{j}^{(i n)} n_{j}^{\prime \prime}\right|_{\partial \Omega^{\prime \prime}} & =\left(F_{p q} F_{p r} n_{q}^{\prime \prime} n_{r}^{\prime \prime}\right)^{1 / 2}\left(J_{0}+C \frac{\mathrm{D} V_{0}}{\mathrm{D} t}\right) \\
\frac{\partial T_{j}^{(e x)}}{\partial y_{j}^{\prime \prime}} & =-\frac{\partial}{\partial x_{i}}\left(F_{i j} \frac{\partial \phi_{1}^{(e x)}}{\partial y_{j}^{\prime \prime}}+\frac{\partial \phi_{0}^{(e x)}}{\partial x_{i}}\right) \quad \text { for } \boldsymbol{y}^{\prime \prime} \in \Omega_{e}, \\
\left.\Theta \varsigma^{(e x)} T_{j}^{(e x)} n_{j}^{\prime \prime}\right|_{\partial \Omega^{\prime \prime}} & =\left(F_{p q} F_{p r} n_{q}^{\prime \prime} n_{r}^{\prime \prime}\right)^{1 / 2}\left(J_{0}+C \frac{\mathrm{D} V_{0}}{\mathrm{D} t}\right)
\end{aligned}
$$

where

$$
T_{j}^{(i n)}=F_{i j}\left(F_{i k} \frac{\partial \phi_{2}^{(i n)}}{\partial y_{k}^{\prime \prime}}+\frac{\partial \phi_{1}^{(i n)}}{\partial x_{i}}\right), \quad T_{j}^{(e x)}=F_{i j}\left(F_{i k} \frac{\partial \phi_{2}^{(e x)}}{\partial y_{k}^{\prime \prime}}+\frac{\partial \phi_{1}^{(e x)}}{\partial x_{i}}\right) .
$$

Applying the conditions (3.16) and (3.17) to $T_{j}^{(i n)}$ and $T_{j}^{(e x)}$, and recalling that $\phi_{1}^{(i n)}$ and $\phi_{1}^{(e x)}$ are given in terms of $\phi_{0}^{(i n)}$ and $\phi_{0}^{(e x)}$ by (3.19), we arrive at the following solvability condition on $\phi_{0}^{(i n)}(\boldsymbol{x}, t)$ and $\phi_{0}^{(e x)}(\boldsymbol{x}, t)$ :

$$
\begin{aligned}
\frac{\partial}{\partial x_{p}}\left(\kappa_{p r}^{(i n)} \frac{\partial \phi_{0}^{(i n)}}{\partial x_{r}}\right) & =-\mathcal{S}\left(J_{0}+C\left(\frac{\partial V_{0}}{\partial t}+(\boldsymbol{U} \cdot \nabla) V_{0}\right)\right), \\
\frac{\partial}{\partial x_{p}}\left(\kappa_{p r}^{(e x)} \frac{\partial \phi_{0}^{(e x)}}{\partial x_{r}}\right) & =\mathcal{S}\left(J_{0}+C\left(\frac{\partial V_{0}}{\partial t}+(\boldsymbol{U} \cdot \nabla) V_{0}\right)\right), \\
V_{0} & =\phi_{0}^{(e x)}-\phi_{0}^{(i n)},
\end{aligned}
$$


where

$$
\mathcal{S}=\frac{1}{\mathcal{V}^{\prime \prime}} \int_{\partial \Omega^{\prime \prime}}\left(F_{l r} F_{l s} n_{r}^{\prime \prime} n_{s}^{\prime \prime}\right)^{1 / 2} d S^{\prime \prime}
$$

and the intracellular and extracellular conductivity tensors are defined by

$$
\begin{aligned}
& \kappa_{p r}^{(i n)}=\frac{\Theta \varsigma^{(i n)}}{\mathcal{V}^{\prime \prime}}\left(\int_{\Omega_{i}^{\prime \prime}} \delta_{p r}+F_{p j} \frac{\partial \Xi_{r}^{(i n)}}{\partial y_{j}^{\prime \prime}} d V^{\prime \prime}\right), \\
& \kappa_{p r}^{(e x)}=\frac{\Theta \varsigma^{(e x)}}{\mathcal{V}^{\prime \prime}}\left(\int_{\Omega_{e}^{\prime \prime}} \delta_{p r}+F_{p j} \frac{\partial \Xi_{r}^{(e x)}}{\partial y_{j}^{\prime \prime}} d V^{\prime \prime}\right),
\end{aligned}
$$

respectively, where $\mathcal{V}^{\prime \prime}=\int_{\Omega_{i}^{\prime \prime}} d V^{\prime \prime}+\int_{\Omega_{e}^{\prime \prime}} d V^{\prime \prime}$ is the volume of the unit cell in the reference domain and $\Xi_{m}^{(i n)}$ and $\Xi_{m}^{(e x)}$ are the solutions to the cell problems (3.20) and (3.21). Equations (3.31)-(3.33) are the widely applied bidomain equations, proposed in [12].

3.3. Comparison with Keener and Panfilov. Keener and Panfilov [16] consider a network of myocytes, and transform to a local curvilinear coordinate system in which one coordinate is aligned with the fiber orientation. This is equivalent to our map $\boldsymbol{x}^{\prime \prime}=\boldsymbol{B}\left(\boldsymbol{x}^{\prime}\right)$. They assume that this curvilinear coordinate system is orthogonal and stretch-free; that is, they consider a geometry in which the transformation of coordinates from the reference configuration (a regular lattice of myocytes) to the actual configuration is everywhere a rotation. In terms of the transformation matrix defined in (3.3) this is equivalent to requiring that the transpose of $F$ be its inverse (i.e., $\left.F_{i j} F_{i k}=\delta_{i k}\right)$. They make a transformation to the reference frame and then perform a multiple-scales analysis analogous to that performed by Neu and Krassowska [17] on a regular lattice of myocytes. However, we believe that [16] contains a couple of typographical errors. In particular, the transformation of derivatives given in (28) is wrong (it should read $\nabla_{x}=T^{T}(x) \nabla_{y}$ ), and the definition of the curvature vector given below (29) is incorrect (it should read $\kappa_{j}=\partial T_{j i} / \partial x_{i}$ ). If we correct for these errors in their analysis, we find that their final result (55) should read (where $F=T^{T}$ )

$$
\frac{\partial}{\partial x_{j}}\left(\sigma_{j k}^{(i n)} \frac{\partial \phi}{\partial x_{k}}\right)=\frac{\epsilon}{\mathcal{V}^{\prime \prime}} \int_{\partial \Omega^{\prime \prime}} I_{m} d S, \quad \frac{\partial}{\partial x_{j}}\left(\sigma_{j k}^{(e x)} \frac{\partial \phi}{\partial x_{k}}\right)=-\frac{\epsilon}{\mathcal{V}^{\prime \prime}} \int_{\partial \Omega^{\prime \prime}} I_{m} d S,
$$

where

$$
\sigma_{j k}^{(i n)}=F_{j i} \Sigma_{i m}^{(i n)} F_{m k}^{T}, \quad \sigma_{j k}^{(e x)}=F_{j i} \Sigma_{i m}^{(e x)} F_{m k}^{T},
$$

and

$$
\Sigma_{i j}^{(i n)}=\frac{\epsilon}{r_{c} \mathcal{V}^{\prime \prime}} \int_{\Omega_{i}^{\prime \prime}}\left(\frac{\partial W_{j}^{(i n)}}{\partial z_{i}}+\delta_{i j}\right) d V, \quad \Sigma_{i j}^{(e x)}=\frac{\epsilon}{r_{c} \mathcal{V}^{\prime \prime}} \int_{\Omega_{e}^{\prime \prime}}\left(\frac{\partial W_{j}^{(e x)}}{\partial z_{i}}+\delta_{i j}\right) d V .
$$

In the case of a rotational transformation, with the property $F_{i j} F_{i k}=\delta_{i k}$ and where we identify $\sigma^{(i n)}$ with $\kappa^{(i n)}, \sigma^{(e x)}$ with $\kappa^{(e x)}, z_{i}$ with $y_{i}^{\prime \prime}, W_{j}^{(i n)}$ with $\Xi_{r}^{(i n)} F_{r j}, W_{j}^{(e x)}$ with $\Xi_{r}^{(e x)} F_{r j}, \epsilon / r_{c}$ with $\varsigma^{(i n)}$ (and $\left.\varsigma^{(e x)}\right)$, and $I_{m}$ with $-\left(C \frac{\partial V}{\partial t}+J\right) /(\epsilon \Theta)$, this is identical to our result (3.31)-(3.33).

The form of $\sigma_{j k}$ above is superficially appealing, since the transformation $F_{i j}$ appears in a natural way in (3.38). However, this hides the fact that, except in the 
particular case that $F_{i j} F_{i k}=\delta_{i k}$, the matrix $F_{i j}$ also appears in the cell problem for $W_{j}$. As noted earlier, the only maps with this property everywhere are global rotations and translations.

3.4. Eulerian interpretation of the conductivity tensors and surface integral. The cell problems (3.20)-(3.21) in reference coordinates involve a relatively unpleasant equation (a general constant-coefficient second-order elliptic operator) on a nice geometry (rectangular periodic). Here we show that these problems can be transformed to an Eulerian frame in which the equation is nice (Laplace's equation) but the geometry is unpleasant (the unit cell is stretched, sheared, and rotated). Although not especially useful for calculating the conductivities, the Eulerian description confirms the intuitively appealing result that the homogenized conductivity tensors at a point may be determined by taking the locally (periodic) structure, extending it to be globally periodic, and homogenizing via multiple scales. The problem with using this method to calculate the conductivities is that the locally periodic structure varies from place to place and is changing in time with the beating of the heart. It is as complicated (if not more complicated) to calculate the effect of this variation on the unit cell as it is to vary the coefficients $F_{i j} F_{i k}$ in (3.20)-(3.21).

The conductivities. We start by transforming the cell problem (3.20)-(3.21) to the Eulerian microscale variable $\boldsymbol{y}=\boldsymbol{x} / \epsilon$. We use the relations that

$$
\frac{\partial}{\partial y_{j}^{\prime \prime}}=F_{j s}^{-1} \frac{\partial}{\partial y_{s}}, \quad n_{j}^{\prime \prime}=F_{j s}^{-1} n_{s}\left(F_{q r} F_{p r} n_{q}^{\prime \prime} n_{r}^{\prime \prime}\right)^{1 / 2}, \quad d V^{\prime \prime}=\left|\operatorname{det}\left(F_{i j}\right)\right| d V
$$

to rewrite (3.35) and (3.36) in the form

$$
\begin{aligned}
& \kappa_{p r}^{(i n)}=\frac{\Theta \varsigma^{(i n)}}{\mathcal{V}}\left(\int_{\Omega_{i}} \delta_{p r}+\frac{\partial \Xi_{r}^{(i n)}}{\partial y_{p}} d V\right), \\
& \kappa_{p r}^{(e x)}=\frac{\Theta \varsigma^{(i n)}}{\mathcal{V}}\left(\int_{\Omega_{e}} \delta_{p r}+\frac{\partial \Xi_{r}^{(e x)}}{\partial y_{p}} d V\right),
\end{aligned}
$$

where $\mathcal{V}=\Omega_{i} \cup \Omega_{e}$ is the volume of the unit cell in the Eulerian frame. The cell problems (3.20) and (3.21) become

$$
\begin{aligned}
& \frac{\partial^{2} \Xi_{\alpha}^{(i n)}}{\partial y_{p} \partial y_{p}}=0 \quad \text { for } \boldsymbol{y} \in \Omega_{i},\left.\quad n_{s} \frac{\partial \Xi_{\alpha}^{(i n)}}{\partial y_{s}}\right|_{\partial \Omega}=-n_{\alpha}, \\
& \frac{\partial^{2} \Xi_{\alpha}^{(e x)}}{\partial y_{p} \partial y_{p}}=0 \quad \text { for } \boldsymbol{y} \in \Omega_{e},\left.\quad n_{s} \frac{\partial \Xi_{\alpha}^{(e x)}}{\partial y_{s}}\right|_{\partial \Omega}=-n_{\alpha},
\end{aligned}
$$

with $\boldsymbol{\Xi}^{(\boldsymbol{i n})}$ and $\boldsymbol{\Xi}^{(\boldsymbol{e x})}$ periodic in $\boldsymbol{y}$. This superficially simpler formulation masks the fact that the domains $\Omega_{i}$ and $\Omega_{e}$ have been sheared, stretched, and rotated by comparison to the rectangular grid of the reference frame, and that this deformation varies in space and may change with time due to the beating of the heart (see Figure 3.1).

The surface integral $\mathcal{S}$. We first note that, for any vector $\mathbf{p}$,

$$
\frac{1}{\mathcal{V}} \int_{\partial \Omega} p_{i} n_{i} d S=\frac{1}{\mathcal{V}} \int_{\Omega} \frac{\partial p_{i}}{\partial y_{i}} d V=\frac{1}{\mathcal{V}^{\prime \prime}} \int_{\Omega^{\prime \prime}} F_{i j} \frac{\partial p_{i}}{\partial y_{j}^{\prime \prime}} d V^{\prime \prime}=\frac{1}{\mathcal{V}^{\prime \prime}} \int_{\partial \Omega^{\prime \prime}} F_{i j} p_{i} n_{j}^{\prime \prime} d S^{\prime \prime}
$$

Then, with $p_{i}=n_{i}$,

$$
\frac{1}{\mathcal{V}^{\prime \prime}} \int_{\partial \Omega^{\prime \prime}} n_{i} F_{i j} n_{j}^{\prime \prime} d S^{\prime \prime}=\frac{1}{\mathcal{V}} \int_{\partial \Omega} n_{i} n_{i} d S
$$


Using (3.4) to reexpress $F_{i j} n_{j}^{\prime \prime}$ as $\left(F_{l r} F_{l s} n_{r}^{\prime \prime} n_{s}^{\prime \prime}\right)^{1 / 2} n_{i}$ in the above formula, it is found that

$$
\mathcal{S}=\frac{1}{\mathcal{V}^{\prime \prime}} \int_{\partial \Omega^{\prime \prime}}\left(F_{l r} F_{l s} n_{r}^{\prime \prime} n_{s}^{\prime \prime}\right)^{1 / 2} d S^{\prime \prime}=\frac{1}{\mathcal{V}} \int_{\partial \Omega} d S
$$

Thus we see that physically $\mathcal{S}$ is the dimensionless surface area of the myocyte within a periodic cell divided by the dimensionless volume of the cell.

4. Summary and conclusions. We have investigated the derivation of the bidomain equations for the electrical activity of the heart from an underlying isotropic microscale model for the electrical activity of cardiac myocytes. This problem has been previously tackled by Krassowska and Neu [17] in the scenario of a uniformly oriented microstructure, and by Keener and Panfilov [16] for a restricted class of nonuniform geometries (global rotations). Our goal was to generalize their approach to the nonuniform geometries encountered in real cardiac tissue and to set up a framework capable of describing electrical activity in deforming tissue such as that encountered in a beating heart. In order to tackle the nonuniform geometry we adapted the multiple-scales method to problems in which the microscale cell problem is almost periodic but exhibits sizeable variations in the shape, size, and orientation of the cells over the macroscale. The result of our multiple-scales analysis was a bidomain model in which the (anisotropic) conductivity tensors vary in space (as the orientation of the myocytes change) and in which the source terms in the bidomain equations, associated with transmembrane current flow and membrane capacitance, vary as a function of the membrane surface area per unit volume. ${ }^{2}$ The anisotropy of the resulting bidomain model arises purely through the geometry of the long thin myocytes and their interconnections.

Our approach involved an adaptation of the multiple-scales technique in which the fast variable is chosen to make the microstructure regular, but the slow variable is the usual Cartesian coordinate. This approach is applicable to other multiple-scales problems in which the microstructure is periodic in some general curvilinear coordinates but in which the homogenized equation is desired in Cartesian coordinates.

There are a number of ways to present the bidomain model that we derive, and we summarize these briefly here. Dropping the subscript 0 for clarity, the dimensionless equations are

$$
\begin{aligned}
\frac{\partial}{\partial x_{p}}\left(\kappa_{p r}^{(i n)} \frac{\partial \phi^{(i n)}}{\partial x_{r}}\right) & =-\mathcal{S}\left(J+C\left(\frac{\partial V}{\partial t}+(\boldsymbol{U} \cdot \nabla) V\right)\right), \\
\frac{\partial}{\partial x_{p}}\left(\kappa_{p r}^{(e x)} \frac{\partial \phi^{(e x)}}{\partial x_{r}}\right) & =\mathcal{S}\left(J+C\left(\frac{\partial V}{\partial t}+(\boldsymbol{U} \cdot \nabla) V\right)\right), \\
V & =\phi^{(e x)}-\phi^{(i n)}
\end{aligned}
$$

where the myocyte surface area-to-volume ratio is

$$
\mathcal{S}=\frac{1}{\mathcal{V}} \int_{\partial \Omega} d S=\frac{1}{\mathcal{V}^{\prime \prime}} \int_{\partial \Omega^{\prime \prime}}\left(F_{l r} F_{l s} n_{r}^{\prime \prime} n_{s}^{\prime \prime}\right)^{1 / 2} d S^{\prime \prime}
$$

in which the tensor $F_{i j}$ is defined by

$$
F_{i j}=\frac{\partial B_{j}}{\partial x_{i}}
$$

\footnotetext{
${ }^{2}$ If the cell surface dilates (increasing $\mathcal{S}$ ) as the result of elastic deformation of the heart, the transmembrane current density $J$ is expected to decrease in proportion to $1 / \mathcal{S}$, since the number of ion channels in the membrane of an individual cell is fixed.
} 
and $\boldsymbol{x}^{\prime \prime}=\boldsymbol{B}(\boldsymbol{x}, t)$ is the mapping from the Eulerian frame to a reference frame in which the cardiac myocytes from a regular periodic array as depicted in Figure 1.2. The intra- and extracellular conductivity tensors, $\kappa_{p r}^{(i n)}$ and $\kappa_{p r}^{(e x)}$, respectively, may be written

$$
\begin{aligned}
& \kappa_{p r}^{(i n)}=\frac{\Theta \varsigma^{(i n)}}{\mathcal{V}^{\prime \prime}}\left(\int_{\Omega_{i}^{\prime \prime}} \delta_{p r}+F_{p j} \frac{\partial \Xi_{r}^{(i n)}}{\partial y_{j}^{\prime \prime}} d V^{\prime \prime}\right), \\
& \kappa_{p r}^{(e x)}=\frac{\Theta \varsigma^{(e x)}}{\mathcal{V}^{\prime \prime}}\left(\int_{\Omega_{e}^{\prime \prime}} \delta_{p r}+F_{p j} \frac{\partial \Xi_{r}^{(e x)}}{\partial y_{j}^{\prime \prime}} d V^{\prime \prime}\right),
\end{aligned}
$$

where $\boldsymbol{\Xi}$ satisfies the cell problems

$$
\begin{gathered}
F_{i j} F_{i k} \frac{\partial^{2} \Xi_{r}^{(i n)}}{\partial y_{j}^{\prime \prime} \partial y_{k}^{\prime \prime}}=0 \quad \text { for } \boldsymbol{y}^{\prime \prime} \in \Omega_{i}^{\prime \prime},\left.\quad F_{i j} F_{i k} n_{j}^{\prime \prime} \frac{\partial \Xi_{r}^{(i n)}}{\partial y_{k}^{\prime \prime}}\right|_{\partial \Omega^{\prime \prime}}=-F_{r q} n_{q}^{\prime \prime}, \\
\Xi_{r}^{(i n)} \quad \text { periodic in } \boldsymbol{y}^{\prime \prime},
\end{gathered}
$$

and

$$
\begin{gathered}
F_{i j} F_{i k} \frac{\partial^{2} \Xi_{r}^{(e x)}}{\partial y_{j}^{\prime \prime} \partial y_{k}^{\prime \prime}}=0 \quad \text { for } \boldsymbol{y}^{\prime \prime} \in \Omega_{e}^{\prime \prime},\left.\quad F_{i j} F_{i k} n_{j}^{\prime \prime} \frac{\partial \Xi_{r}^{(e x)}}{\partial y_{k}^{\prime \prime}}\right|_{\partial \Omega^{\prime \prime}}=-F_{r q} n_{q}^{\prime \prime}, \\
\Xi_{r}^{(e x)} \quad \text { periodic in } \boldsymbol{y}^{\prime \prime} .
\end{gathered}
$$

Alternatively, they may be written as

$$
\begin{aligned}
& \kappa_{p r}^{(i n)}=\frac{\Theta \varsigma^{(i n)}}{\mathcal{V}^{\prime \prime}} F_{p j}\left(\int_{\Omega_{i}^{\prime \prime}} \delta_{j k}+\frac{\partial W_{k}}{\partial y_{j}^{\prime \prime}} d V^{\prime \prime}\right) F_{k r}^{-1}, \\
& \kappa_{p r}^{(e x)}=\frac{\Theta \varsigma^{(e x)}}{\mathcal{V}^{\prime \prime}} F_{p j}\left(\int_{\Omega_{e}^{\prime \prime}} \delta_{j k}+\frac{\partial W_{k}}{\partial y_{j}^{\prime \prime}} d V^{\prime \prime}\right) F_{k r}^{-1},
\end{aligned}
$$

where $\boldsymbol{W}$ satisfies the cell problems

$$
\begin{gathered}
F_{i j} F_{i k} \frac{\partial^{2} W_{r}}{\partial y_{j}^{\prime \prime} \partial y_{k}^{\prime \prime}}=0 \quad \text { for } \boldsymbol{y}^{\prime \prime} \in \Omega_{i}^{\prime \prime},\left.\quad F_{i j} F_{i k} n_{j}^{\prime \prime} \frac{\partial W_{r}}{\partial y_{k}^{\prime \prime}}\right|_{\partial \Omega^{\prime \prime}}=-F_{p q} F_{p r} n_{q}^{\prime \prime}, \\
W_{r} \quad \text { periodic in } \boldsymbol{y}^{\prime \prime},
\end{gathered}
$$

and

$$
F_{i j} F_{i k} \frac{\partial^{2} W_{r}}{\partial y_{j}^{\prime \prime} \partial y_{k}^{\prime \prime}}=0 \quad \text { for } \boldsymbol{y}^{\prime \prime} \in \Omega_{e}^{\prime \prime},\left.\quad F_{i j} F_{i k} n_{j}^{\prime \prime} \frac{\partial W_{r}}{\partial y_{k}^{\prime \prime}}\right|_{\partial \Omega^{\prime \prime}}=-F_{p q} F_{p r} n_{q}^{\prime \prime}
$$

$W_{r} \quad$ periodic in $\boldsymbol{y}^{\prime \prime}$.

In both cases the geometry of the unit cell is rectangular since it is described in terms of the reference variable $\boldsymbol{y}^{\prime \prime}$.

Alternatively we may write

$$
\begin{aligned}
& \kappa_{p r}^{(i n)}=\frac{\Theta \varsigma^{(i n)}}{\mathcal{V}}\left(\int_{\Omega_{i}} \delta_{p r}+\frac{\partial \Xi_{r}^{(i n)}}{\partial y_{p}} d V\right), \\
& \kappa_{p r}^{(e x)}=\frac{\Theta \varsigma^{(i n)}}{\mathcal{V}}\left(\int_{\Omega_{e}} \delta_{p r}+\frac{\partial \Xi_{r}^{(e x)}}{\partial y_{p}} d V\right),
\end{aligned}
$$


where $\boldsymbol{\Xi}$ satisfies the Eulerian cell problems

$$
\begin{aligned}
& \frac{\partial^{2} \Xi_{\alpha}^{(i n)}}{\partial y_{p} \partial y_{p}}=0 \quad \text { for } \boldsymbol{y} \in \Omega_{i},\left.\quad n_{s} \frac{\partial \Xi_{\alpha}^{(i n)}}{\partial y_{s}}\right|_{\partial \Omega}=-n_{\alpha}, \\
& \frac{\partial^{2} \Xi_{\alpha}^{(e x)}}{\partial y_{p} \partial y_{p}}=0 \quad \text { for } \boldsymbol{y} \in \Omega_{e},\left.\quad n_{s} \frac{\partial \Xi_{\alpha}^{(e x)}}{\partial y_{s}}\right|_{\partial \Omega}=-n_{\alpha} \text {. }
\end{aligned}
$$

In this case the unit cell has been sheared, stretched, and rotated by comparison to rectangular Cartesian coordinates (through the map $\boldsymbol{x}^{\prime \prime}=\boldsymbol{B}(\boldsymbol{x}, t)$ ), which is where the local geometry of the microstructure comes into play.

The presence of the tensor $F_{i j}$ in the cell problems above is unfortunate, since it means that we cannot solve a single cell problem to find the effective conductivities (remember that $F_{i j}$ is a function of $\boldsymbol{x}$ ). Instead a numerical procedure such as the heterogeneous multiscale method [9] would need to be employed, with a cell problem solved for each element of a finite element implementation of the bidomain equations.

This leads to the natural question of whether an ad hoc approximation could be made, as is usual in the literature $[5,14]$, in which the conductivities are assumed to be of the form

$$
\kappa_{p r}^{(i n)}=F_{p j} \Sigma_{j k}^{(i n)} F_{k r}^{-1}, \quad \kappa_{p r}^{(e x)}=F_{p j} \Sigma_{j k}^{(e x)} F_{k r}^{-1}
$$

with $\Sigma_{j k}^{(i n)}$ and $\Sigma_{j k}^{(e x)}$ constant and independent of position and where $F_{p j}(\boldsymbol{x})$ is a rotation matrix aligned to account for the local orientation of the myocytes. Such an approximation may not be so bad, since the fact that the conductivites along a fiber and across a fiber are so different may mean that the rotation of the fiber is the dominant effect, with shear and stretch being secondary. It will be interesting to solve the cell problems (4.7)-(4.8) or (4.11)-(4.12) numerically for representative geometries to evaluate the validity of this assumption. For a general deformation this approximation will need to be combined with an extraction of the relevant rotational component of $F_{i j}$ by decomposing $\boldsymbol{B}$ into sequential stretches, shear across the fiber direction, and rotations.

We emphasize that our method is general and is able to account both for (i) cardiac structures in which the intermyocyte electrical connections in the directions perpendicular to the long axis of the myocytes are similar and for (ii) orthotropic structures in which the myocytes are arranged in sheet-like structures with relatively weak electrical connections between sheets (see, e.g., [14]) but with much stronger connections between adjacent myocytes in the same sheet. Evidence for such orthotropic conductivities was given recently in [6].

Finally, we note that there is evidence that inexcitable fibroblasts, which make up a significant fraction of cardiac tissue, are electrically connected to cardiac myocytes via functional gap junctions [7]. Although we have not accounted for this explicitly, it would be straightforward to generalize our method to do so.

\section{REFERENCES}

[1] L. Barr and E. Jacobson, The spread of current in the electrical syncytia, in Physiology of Smooth Muscle, E. Bulbring and M. F. Shuba, eds., Raven Press, New York, 1976.

[2] G. W. Beeler And H. Reuter, Reconstruction of the action potential of ventricular myocardial fibres, J. Physiol., 268 (1977), pp. 177-210. 
[3] Y. Bourgault, Y. Coudiére, And C. Pierre, Existence and uniqueness of the solution for the bidomain model used in cardiac electrophysiology, Nonlinear Anal. Real World Appl., 10 (2009), pp. 458-482.

[4] M. R. Boyett, J. E. Frampton, And M. S. Kirby, The length, width and volume of isolated rat and ferret ventricular myocytes during twitch contractions and changes in osmotic strength, Exper. Physiol., 76 (1991), pp. 259-270.

[5] M. Buist, G. Sands, P. Hunter, and A. Pullan, A deformable finite element derived finite difference methods for cardiac activation problems, Ann. Biomed. Eng., 31 (2003), pp. $557-588$.

[6] B. J. Caldwell, M. L. Trew, G. B. Sands, D. A. Hooks, I. J. LeGrice, and B. H. Smaill, Three distinct directions of intramural activation reveal nonuniform side-to-side electrical coupling of ventricular myocytes, Circ. Arrhythm. Electrophysiol., 2 (2009), pp. 433-440.

[7] P. Camelliti, C. R. Green, I. LeGrice, and P. Kohl, Fibroblast network in rabbit sinoatrial node: Structural and functional identification of homogeneous and heterogeneous cell coupling, Circ. Res., 94 (2004), pp. 828-835.

[8] D. Durrer, R. Th. Van Dam, G. E. Freud, M. J. Janse, F. L. Meijler, and R. C. Arzbaecher, Total excitation of the isolated human heart, Circulation, 41 (1970), pp. 899-912.

[9] W. E, B. Engquist, X. Li, W. Ren, and E. Vanden-Eijnden, Heterogeneous multiscale methods: A review, Comm. Comput. Phys., 2 (2007), pp. 367-450.

[10] I. R. Efimov, V. P. Nikolski, and G. Salama, Optical imaging of the heart, Circulation Res., 95 (2004), pp. 21-33.

[11] R. S. Eisenberg, V. Barcillon, and R. T. Mathias, Electrical properties of spherical syncytia, Biophys. J., 25 (1979), pp. 151-180.

[12] C. Henriquez, Simulating the electrical behavior of cardiac tissue using the bidomain model, Crit. Rev. Biomed. Eng., 21 (1993), pp. 1-77.

[13] A. L. Hodghin And A. F. Huxley, A quantitative description of membrane current and its application to conduction and excitation in nerve, J. Physiol., 117 (1952), pp. 500-544.

[14] D. A. Hooks, M. L. Trew, B. J. Caldwell, G. B. Sands, I. J. LeGrice, and B. H. Smaill, Laminar arrangement of ventricular myocytes influences electrical behavior of the heart, Circ. Res., 101 (2007), pp. e103-e112.

[15] Y. Jiang, K. Pandya, O. Smithies, and E. W. Hsu, Three-dimensional diffusion tensor microscopy of fixed mouse hearts, Magnetic Resonance Med., 52 (2004), pp. 453-460.

[16] J. P. Keener And A. V. Panfilov, A biophysical model of defibrillation in cardiac tissue, Biophys. J., 71 (1996), pp. 1335-1345.

[17] W. Krassowska and J. C. Neu, Homogenization of syncytial tissues, Crit. Rev. Biomed. Eng., 21 (1993), pp. 137-199.

[18] J. R. Levick, An Introduction to Cardiovascular Physiology, Arnold, London, 2000.

[19] S. E. Litwin, S. E. Katz, J. P. Morgan, and P. S. Douglas, Serial echocardiographic assessment of left ventricular geometry and function after large myocardial infarction in the rat, Circ. Res., 89 (1994), pp. 345-354.

[20] C. H. Luo And Y. Rudy, A dynamic model of the cardiac ventricular action potential. I. Simulations of ionic currents and concentration changes, Circ. Res., 74 (1994), pp. 10711096.

[21] W. T. Miller and D. B. Gesolowitz, Simulation studies of the electrocardiogram. I. The normal heart, Circ. Res., 43 (1978), pp. 301-315.

[22] D. Noble And Y. Rudy, Models of ventricular cardiac action potentials: Iteractive interaction between experiment and simulation, Phil. Trans. R. Soc. Lond. A, 359 (2001), pp. 11271142 .

[23] G. Olivetti, M. Melissari, J. M. Capasso, and P. Anversa, Cardiomyopathy of the aging human heart. Myocyte loss and reactive cellular hypertrophy, Circ. Res., 68 (1991), pp. $1560-1568$.

[24] R. Penland, D. M. Harrild, and C. S. Henriquez, Modeling impulse propagation and extracellular potential distributions in anisotropic cardiac tissue using a finite volume element discretization, Comput. Visual Sci., 4 (2002), pp. 215-226.

[25] M. Pennacchio, G. Savaré, and P. C. Franzone, Multiscale modeling for the bioelectric activity of the heart, SIAM J. Math. Anal., 37 (2006), pp. 1333-1370.

[26] A. Peskoff, Electrical potential in three-dimensional electrically syncytial tissues, Bull. Math. Biophys., 41 (1979), pp. 163-181.

[27] P. A. Poole-Wilson, The dimensions of human cardiac myocytes; Confusion caused by methodology and pathology, J. Mol. Cell. Cardiol., 27 (1995), pp. 863-865. 
[28] G. Richardson, A multiscale approach to modelling electrochemical processes occurring across the cell membrane with application to transmission of action potentials, Math. Med. Biol., 26 (2009), pp. 201-224.

[29] H. I. SAlEhEen AND K. T. NG, A new three-dimensional finite-difference bidomain formulation for inhomogeneous anisotropic cardiac tissues, IEEE Trans. Bio. Eng., 45 (1998), pp. 1525.

[30] P. Schmid, T. Jaermann, P. Boesiger, P. F. Niederer, P. P. Lunkenheimer, C. W. Cryer, AND R. H. ANDERSON, Ventricular myocardial architecture as visualised in postmortem swine hearts using magnetic resonance, Eur. J. Cardiothorac. Surg., 27 (2005), pp. 468474.

[31] M. S. Spach, W. T. Miller, P. C. Dolber, J. M. Kootskey, J. R. Sommer, and C. E. Mosher, The functional role of structural complexities in the propagation of depolarization in the atrium of the dog, Circ. Res., 50 (1982), pp. 175-191.

[32] M. L. Trew, B. J. Caldwell, G. B. Sands, D. A. Hooks, D. C.-S. Tai, T. M. Austin, I. J. LeGrice, A. J. Pullan, And B. H. Smaill, Cardiac electrophysiology and tissue structure: Bridging the scale gap with a joint measurement and modelling paradigm, Exper. Physiol., 91 (2006), pp. 355-370.

[33] M. Veneroni, Reaction-diffusion systems for the macroscopic bidomain model of the cardiac electric field, Nonlinear Anal. Real World Appl., 10 (2009), pp. 849-868.

[34] A. Xu And M. R. Guevara, Two forms of spiral-wave reentry in an ionic model of ischemic ventricular myocardium, Chaos, 8 (1998), pp. 157-174. 